\title{
Dinámica de una red integral de prestadores de servicios de salud (RIPSS)*
}

\author{
Dynamics of a Comprehensive Network of Health Service Suppliers (RIPSS) \\ Dinâmica de uma rede integral de prestadores de serviços de saúde (RIPSS)
}

Raúl Andrés Tabarquino Muñoz ${ }^{\text {a }}$

Universidad del Valle, Colombia

ORCID: http://orcid.org/0000-0002-7866-1875

DOI: https://doi.org/10.11144/Javeriana.rgps17-34.drip

Dora Marcela Pulgarin Henao

Fecha de recepción: 29 Junio 2017

Agencia Aduanera Servadi, Colombia

ORCID: http://orcid.org/0000-0002-9228-7576

Albert Ferney Giraldo Varón

Promoción y Relaciones Corporativas del Sena, Colombia

ORCID: http://orcid.org/0000-0002-4439-2582

\section{Resumen:}

La Política Pública de Atención Integral en Salud del año 2016 en Colombia establece el esquema de redes integrales de prestadores de servicios de salud (RIPSS), el cual dinamiza demandas y recursos proyectados, personal calificado e infraestructura en red. El análisis de este nuevo esquema de prestación de servicios de salud parte de un enfoque metodológico mixto con aplicación de análisis de redes, apropiando el caso de la red de salud del oriente del municipio de Santiago de Cali, por la existencia de avances y aportes en la aplicación del concepto de redes integradas de servicios de salud (RISS) y logística integral. Los resultados de la investigación aportan teórica, analítica y metodológicamente que la interdependencia, interrelación y coordinación entre actores en una red integral de prestadores de servicios de salud (RIPSS) puede ser simulada de manera estructural y posicional.

Palabras clave: planificación y administración en salud, políticas públicas de salud, integralidad en salud, gestión en salud, atención primaria de salud, análisis de redes.

\section{Abstract:}

The Colombian public policy 'Comprehensive Health Care 2016' sets an all-encompassing network scheme for health service suppliers (RIPSS, by its Spanish acronym), which provides a better dynamics between projected demands and resources, qualified personnel, and networked infrastructure. The analysis of this new health service provision scheme is based on a combined methodological approach with a network analysis application. This work addresses specifically the case of the West health service network in Santiago de Cali, as there have been advancements and contributions to implement the concept of integrated health service networks (RISS) and comprehensive logistics. The research results indicate theoretically, analytically and methodologically that the interdependence, interrelation, and coordination between the actors in a comprehensive network of health service suppliers (RIPSS) can be simulated structurally and positionally.

Keywords: health planning and administration, health public policies, comprehensive health care, health management, primary health care, network analysis.

\section{Resumo:}

A Política Pública de Atenção Integral a Saúde do ano 2016 na Colômbia estabelece o esquema de redes integrais de prestadores de serviços de saúde (RIPSS), a qual dinamiza demandas e recursos projetados, pessoal qualificado e infraestrutura em rede. A análise desse novo esquema de prestação de serviços de saúde parte de uma abordagem metodológica mista com aplicação de análise de redes, apropriando o caso da rede de saúde do oriente do município de Santiago de Cali, pela existência de avanços e contribuições na aplicação do conceito de redes integradas de serviços de saúde (RISS) e logística integral. Os resultados da pesquisa contribuem teórica, analítica e metodologicamente ao fato da interdependência, inter-relação e coordenação entre atores em uma rede integral de prestadores de serviços de saúde (RIPSS) puder ser simulada de maneira estrutural e posicional.

Palavras-chave: planificação e administração em saúde, políticas públicas de saúde, integralidade em saúde, gestão em saúde, atenção primária de saúde, análise de redes.

\footnotetext{
${ }^{a}$ Autor de correspondencia. Correo electrónico: ranta0831@gmail.co
} 


\section{Introducción}

La salud en Colombia evidencia fragmentación de servicios de salud y aseguramiento, por la centralidad y concentración de recursos en organizaciones públicas y privadas. En las dos últimas décadas han aplicado de manera marginal acciones promocionales y preventivas por razones de lógicas mercantiles que generan barreras de acceso al servicio, ineficiencia en la utilización de los recursos disponibles y baja calidad técnica y operativa de los procesos logísticos y administrativos. Debido a esta situación problemática, se definido un marco regulatorio que propone esquemas de prestación del servicios de salud basados en las dinámica de redes (RAS) y la atención primaria en salud (APS) en Colombia, con el fin de consolidar estructuras de atención integrada, integral y continua en sector de la salud, que necesariamente deben articular la logística integral como un área estratégica de las IPS y las EPS, validando e implementando un esquema de gestión en tiempos, escalas y resultados, con el fin de prestar un servicio eficaz y eficiente con un impacto directo, positivo e incremental en el paciente-usuario.

Uno de estos esquemas son las redes integrales de prestadores de servicios de salud (RIPSS), que se instauran como línea en la Política Pública de Atención Integral en Salud en el año 2016 en Colombia y se regulan e implementa por la Resolución 1441 del 21 de abril del 2016 [1], la cual define en su artículo 14 un conjunto de actores involucrados y responsables de manera directa para la habilitación de las RIPSS, que se categorizan en un esquema de política pública como actores reguladores, actores regulados y actores no regulados, como lo muestra la tabla 1.

\section{TABLA 1}

Actores que participan en la Red Integral de Prestadores de Servicios de Salud (RIPSS)

\begin{tabular}{|c|c|}
\hline Actores reguladores & Actores regulados \\
\hline $\begin{array}{l}\text { Ministerio de Salud y Protección Social } \\
\text { Secretarías de Salud } \\
\text { Superintendencia Nacional de Salud } \\
\text { Consejos territoriales de seguridad social en } \\
\text { salud }\end{array}$ & $\begin{array}{l}\text { Empresas promotoras de salud (EPS) } \\
\text { Instituciones prestadoras de servicios de } \\
\text { salud (IPS): hospitales, centros de salud, } \\
\text { puestos de salud, clínicas y laboratorios }\end{array}$ \\
\hline \multicolumn{2}{|c|}{ Actores no regulados } \\
\hline \multicolumn{2}{|c|}{ Proveedores } \\
\hline
\end{tabular}

Fuente: elaboración propia a partir de la Resolución 1441 de 2016.

Este nuevo modelo o esquema de prestación de servicios en salud conlleva como objetivo de investigación analizar el esquema propuesto de RIPSS en Colombia por la Política Pública de Atención Integral en Salud del año 2016. En ese sentido, es importante indagar con relación a cuál es la dinámica estructural y posicional de un esquema de RIPSS, a partir del conjunto de actores que define la Resolución 1441 del 21 de abril del 2016 [1] en su artículo 14 para la habilitación de las RIPSS.

La operativización del objetivo de investigación y la respuesta de la pregunta de investigación exigen la definición de RIPSS, la cual, según el Ministerio de Salud y Protección Social [1], es:

“ [...] el conjunto articulado de prestadores de servicios de salud u organizaciones funcionales de servicios de salud, públicos y privados; ubicados en un ámbito territorial definido de acuerdo con las condiciones de operación del Modelo integral de Atención en Salud (MIAS), con una organización funcional que comprende un componente primario y un componente complementario; bajo los principios de disponibilidad, aceptabilidad, accesibilidad y calidad al igual que los mecanismos 
requeridos para la operación y gestión de la prestación de servicios de salud, con el fin de garantizar el acceso y la atención oportuna, continua, integral, resolutiva a la población, contando con los recursos humanos, técnicos, financieros y físicos para garantizar la gestión adecuada de la atención, así como de los resultados en salud."

Este concepto obliga a diferenciar entre redes integradas y redes integrales de salud, lo que explica la Corte Constitucional de Colombia en la Sentencia C-313 de 2014. De acuerdo con la Corporación, la primera se refiere a los sistemas interinstitucionales de salud como entidades u organizaciones que comparten una dinámica en red de orden funcional y la segunda a su estructura en red, que aglutina entidades $u$ organizaciones con tecnología que garantizan la cobertura y el acceso en materia de salud.

Esta diferenciación permite profundizar y organizar el abordaje teórico y conceptual en redes de atención en salud (RAS), con el fin de explicar su relación con la atención primaria en salud (APS) y la diferencia entre redes integradas y redes integrales de salud. La Organización Panamericana de Salud (OPS) publicó un documento técnico en el año 2013 que aborda estas diferencias teóricas y conceptuales y que fue escrito por el investigador Eugenio Vilaça Mendes [2]. Allí se explica que las RAS son: i) "arreglos organizativos de acciones y servicios de salud, de diferentes densidades tecnológicas, que integradas a través de sistemas técnico, logístico y de gestión, buscan garantizar la integralidad del cuidado"; y ii) "organizaciones poliárquicas de conjuntos de servicios de salud, vinculados entre sí por una misión única, por objetivos comunes y por una acción cooperativa e interdependiente, que permiten ofertar una atención continua e integral a determinada población, coordinada por la atención primaria de salud - proporcionada en el momento oportuno, en el lugar apropiado, al costo adecuado, con la calidad necesaria, de forma humanizada y con equidad- y con responsabilidades sanitaria y económica y generando valor para la población”.

Mendes [2] explica que las RAS en su estructura operacional involucran de manera obligada un sistema logístico - tarjeta de identificación de las personas usuarias, historia clínica, sistemas regulados de acceso a la atención y sistemas de transporte en salud - y un sistema de apoyo — sistema de apoyo diagnóstico y terapéutico, sistema de asistencia farmacéutica y sistema de información en salud, como se aprecia en la figura 1- que articula la APS. Esta última se define como:

“La atención esencial de salud, basada en métodos prácticos, científicamente sólidos y socialmente aceptables y tecnologías universalmente accesibles para los individuos y familias de la comunidad, mediante su plena participación y a un costo al que las comunidades y los países puedan acceder, en todas las etapas de su desarrollo, con un espíritu de autorresponsabilidad y autodeterminación. Ella forma parte integrante del sistema de atención de salud del cual representa su función central y el principal foco de desarrollo económico y social de la comunidad. Constituye el primer contacto de individuos, familias y comunidades con el sistema de atención de salud, trayendo los servicios de salud lo más próximo posible a los lugares de vida y trabajo de las personas y significa el primer elemento de un proceso continuo de atención [3].” 


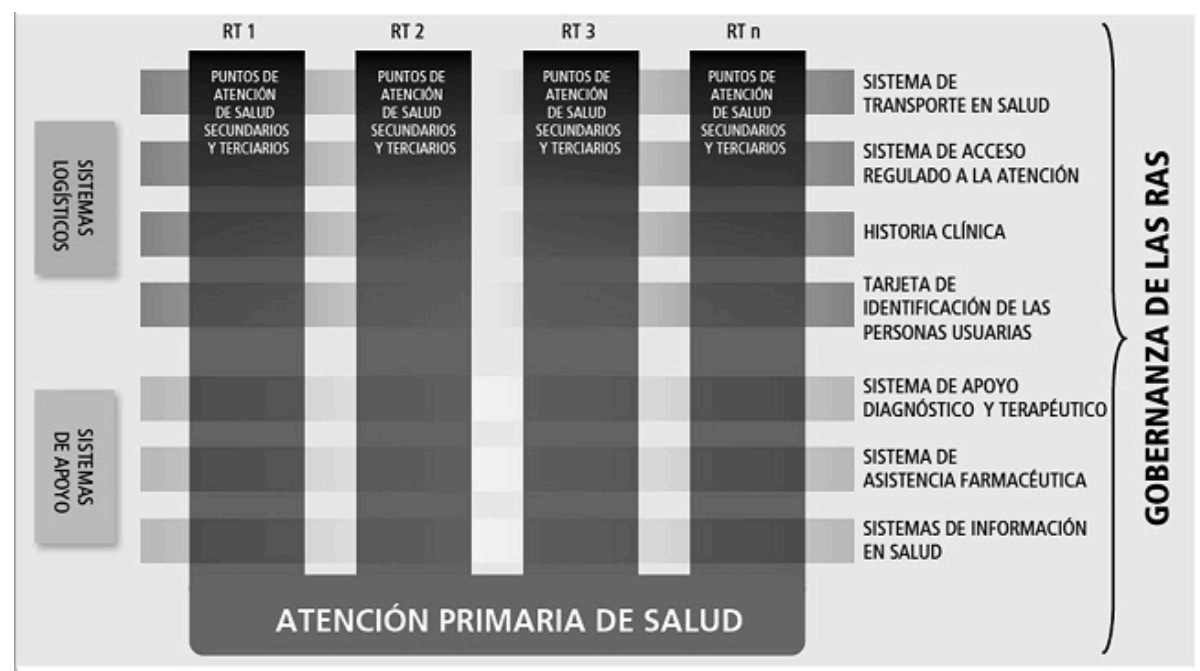

FIGURA 1

La estructura operacional de las redes de atención de salud Fuente: [2].

Este sistema de apoyo y sistema logístico de las RAS se plasma como línea de política pública en salud mediante el concepto de redes integradas de servicios de salud (RISS) que reposa en dos documentos técnicos publicados por la OPS y la Organización Mundial de la Salud (OMS) en los años 2007 (documento de posición OPS/OMS) y 2010 (RISS, conceptos y hoja de ruta), en cuanto a la disertación sobre la renovación de la APS en las Américas [3, 4].

La OPS/OMS [4] define las RISS como:

“Las Redes Integradas de Servicios de Salud, o Sistemas Organizados de Servicios de Salud, o Sistemas Clínicamente Integrados, u Organizaciones Sanitarias Integradas, pueden definirse como: una red de organizaciones que presta, o hace los arreglos para prestar, servicios de salud equitativos e integrales a una población definida, y que está dispuesta a rendir cuentas por sus resultados clínicos y económicos y por el estado de salud de la población a la que sirve."

Otros autores las definen como el conjunto de actores que son por naturaleza organizaciones debidamente constituidas y que adquieren responsabilidad jurídica respondiendo a una jerarquía, coordinación, promoción de programas y servicios continuos a una población ubicada en un territorio especifico, generando impacto de manera directa en aspectos fiscales, clínicos y psicosociales de los beneficiados $[5,6,7,8,9,10$, $11,12,13,14,15]$.

Las RISS se insertan en sector de la salud en Colombia mediante la Ley 1438 de 2011 —reforma del Sistema General de Seguridad Social en Salud- [16], en la que se les define como "el conjunto de organizaciones o redes que prestan servicios o hacen acuerdos para prestar servicios de salud individuales y/o colectivos, más eficientes, equitativos, integrales, continuos a una población definida, dispuesta conforme a la demanda”, articuladas por entidades territoriales en coordinación con EPS mediante los consejos territoriales de salud y con los objetivos de: i) no vulnerar la autonomía de los actores que participan en la red y ii) brindar un servicio de salud preciso, oportuno, pertinente, eficaz, costofectivo, de calidad, con optimización de recursos e impacto clínico en la población.

Este concepto de RISS lo tiene en cuenta la Ley 1751 de 2015 - que regula el derecho fundamental a la salud- [17] en cuanto a la introducción del concepto de integralidad:

“Artículo $8^{\circ}$. La integralidad. Los servicios y tecnologías de salud deberán ser suministrados de manera completa para prevenir, paliar o curar la enfermedad, con independencia del origen de la enfermedad o condición de salud, del sistema de provisión, cubrimiento o financiación definido por el legislador. No podrá fragmentarse la responsabilidad en la prestación de un servicio de salud específico en desmedro de la salud del usuario. En los casos en los que exista duda sobre el alcance de un 
servicio o tecnología de salud cubierto por el Estado, se entenderá que este comprende todos los elementos esenciales para lograr su objetivo médico respecto de la necesidad específica de salud diagnosticada."

El concepto de integralidad se plasma mediante la Resolución 429 del 17 de febrero de 2016 [18], que adopta la Política de Atención Integral en Salud y que establece el concepto de RIPSS a partir del ámbito territorial de operación de la red, en cuanto a: i) necesidades de la población; ii) caracterización de riesgo de las aseguradoras para la población afiliada; iii) requerimientos de las rutas integrales de atención en salud (RIAS) y iv) los resultados y datos de la definición de grupos poblacionales y grupos de riesgo. Y, a su vez, debe cumplir con el componente primario y complementario de prestación en forma de red, como se evidencia en la figura 1.

La Política de Atención Integral en Salud [19] reza, como lo evidencia la figura 2, que la conformación, organización y gestión de las RIPSS deben cumplir con los siguientes objetivos: i) caracterizar y estimar las necesidades de la población, teniendo como criterio la capacidad instalada disponible; ii) definir el portafolio de servicios que se puede cumplir en el territorio, de acuerdo con el criterio de los servicios existentes y habilitados; iii) definir procesos, procedimientos, mecanismos administrativos y asistenciales.

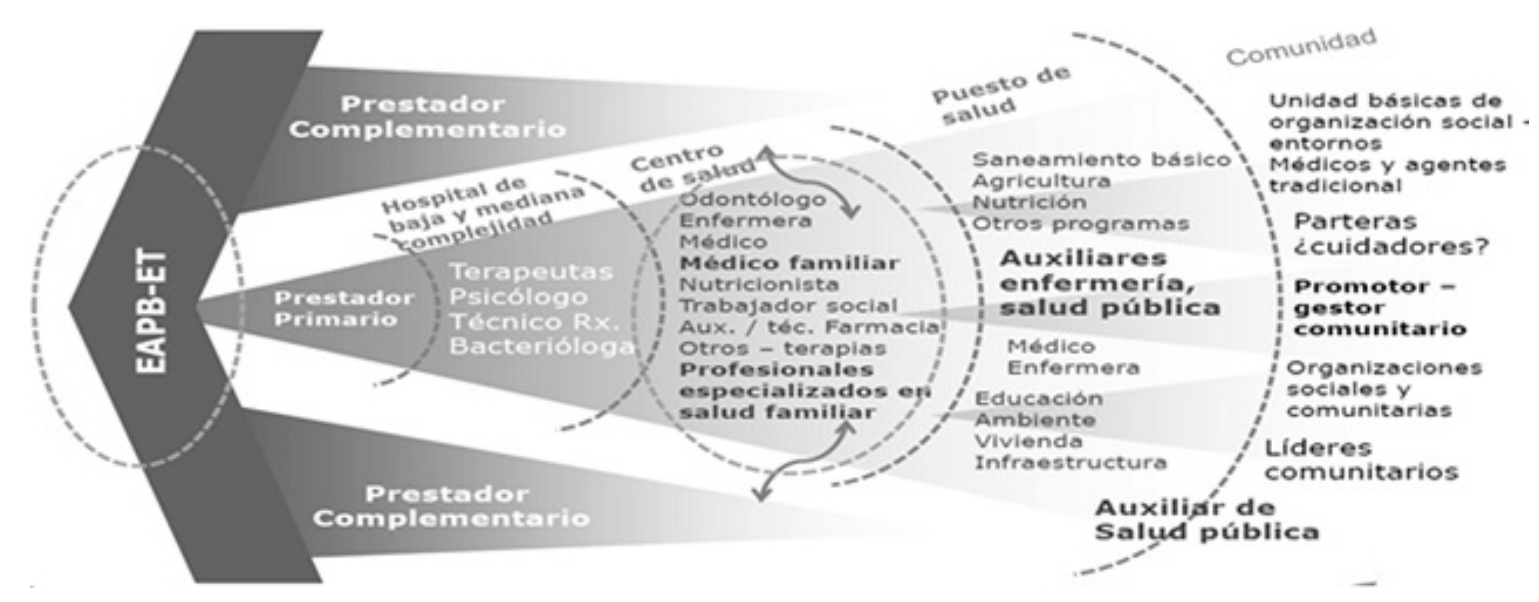

FIGURA 2

Componente primario y complementario de prestación en forma de red Fuente: [19]. 
El esquema propuesto implica interdependencia, interrelación y coordinación de actores públicos, privados y mixtos en un territorio, con tecnología y competencias especializadas para enfermedades, incorporación de servicios de complejidad y pertinencia e incidencia socialcultural en el territorio abarcado e intervenido por la RIPSS, como se esboza en la figura 3.

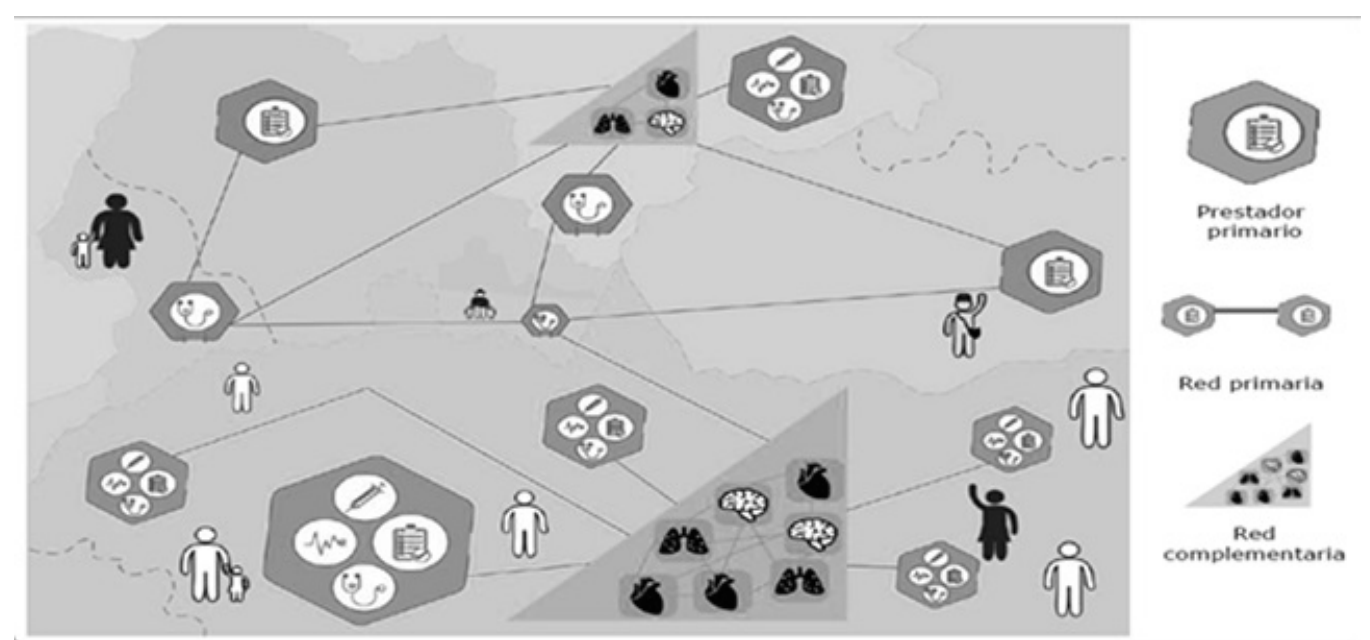

FIGURA 3

Dinámica y estructura redes integrales de prestadores de servicios de salud Fuente: [19].

Para la existencia de interdependencia, interrelación y coordinación de actores públicos, privados y mixtos en un territorio con tecnología y competencias, la Política de Atención Integral en Salud en Colombia establece unas condiciones mínimas [19] que se pueden evidenciar, plasmar y cumplir con unas indicaciones mínimas. Estas se organizan en atributos de funcionamiento independientes del modelo organizacional acotado por los actores en los diferentes contextos y territorios establecidos por la OPS [4] y de conformidad con la premisa de que el esquema de atención en salud primaria mediante redes debe ser una estrategia para garantizar el acceso, la eficacia y la eficiencia en la prestación de servicios de salud en un territorio, con la incorporación de herramientas de logística integral que permitan consolidar los atributos mínimos establecidos por la OPS, como se evidencia en la tabla 2. De no ser así, se aplican redes de atención en servicios de salud fragmentadas o parcialmente integradas [13]. 
TABLA 2

Atributos mínimos de una RIPSS a partir de los atributos mínimos establecidos por la OPS [4] con la incorporación de herramientas logísticas de Pulgarín [13]

\begin{tabular}{|c|c|c|}
\hline $\begin{array}{l}\text { Ambitos } \\
\text { de } \\
\text { abordaje }\end{array}$ & Atributos OPS (4) & $\begin{array}{l}\text { Herramientas Logisticas } \\
\text { administrattvas Pulgartn (13) }\end{array}$ \\
\hline \multirow{6}{*}{ 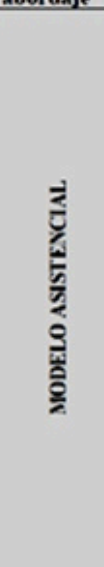 } & $\begin{array}{l}\text { 1. Población y territorio a cargo, definido y amplio conocimiento de sus } \\
\text { necesidades y preferencias en cuestiones de salud, que determinan ta oferta de } \\
\text { servicios de salud. }\end{array}$ & $\begin{array}{l}\text { - Sistema CRM }- \text { Cloud } \\
\text { computing. }\end{array}$ \\
\hline & $\begin{array}{l}\text { 2. Una extensa red de establecimientos de salud que presta servicios de } \\
\text { promocion. prevencion. diagnóstico, tratamiento. gestion de enfermedades. } \\
\text { rehabilitacion y cuidados paliativos, y que integra los programas focalizados } \\
\text { en enfermedades, riesgos y poblaciones especificas, los servicios de salud } \\
\text { personales y los servicios de salud publica. }\end{array}$ & $\begin{array}{l}\text { - Implementación sistema CRM - } \\
\text { Segmentación }\end{array}$ \\
\hline & $\begin{array}{l}\text { 3. Un primer nivel de atencion multidisciplinario que abarca a toda la } \\
\text { poblacion y sirve como puerta de entrada al sistema. que intega y coordina la } \\
\text { atención de salud. además de satisfacer la mayor parte de las necesidades de } \\
\text { salud de la población. }\end{array}$ & - Telemedicina Intrahospitaria. \\
\hline & $\begin{array}{l}\text { 4. Prestación de servicios especializados en el lugar mas apropiado. que se } \\
\text { ofrecen de preferencia en entormos extra hospitalarios. }\end{array}$ & - Telemedicina Extrahospitalia. \\
\hline & $\begin{array}{l}\text { 5. Existencia de mecanismos de coordinación asistencial a lo largo de todo el } \\
\text { continuo de los servicios de salud. }\end{array}$ & $\begin{array}{l}\text { - Unidades lectoras de código de } \\
\text { barras. lecnologias de } \\
\text { identificación. } \\
\text { - Sistema de transporte satelital }\end{array}$ \\
\hline & $\begin{array}{l}\text { 6. Atención de salud centrada en la persona, la familia y la comunidad, a partir } \\
\text { de las particularidades culturales y de género. y los niveles de diversidad de la } \\
\text { población. }\end{array}$ & - Sistema CRM - Pagina Web. \\
\hline \multirow{3}{*}{ 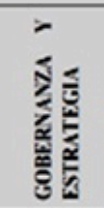 } & 7. Un sistema de dirección unico para toda la red. & $\begin{array}{l}\text { - Cuadro de mando integral } \\
\text { (CMI) }\end{array}$ \\
\hline & 8. Participación social amplia. & - Sistema CRM - Redes sociales \\
\hline & $\begin{array}{l}\text { 9. Acción intersectorial y abordaje de los determinantes de salud y la equidad } \\
\text { en salud. }\end{array}$ & $\begin{array}{l}\text { - Transferencia electrónica de } \\
\text { datos (EDI) }\end{array}$ \\
\hline \multirow{4}{*}{ 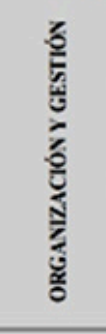 } & $\begin{array}{l}\text { 10. Gestion integrada de los sistemas de apoyo administrativo, clinico y } \\
\text { logistico. }\end{array}$ & $\begin{array}{l}\text { - Cuadro de mando Integral } \\
\text { (CMI) }\end{array}$ \\
\hline & $\begin{array}{l}\text { 11. Recursos humanos suficientes, competentes, comprometidos y valorados } \\
\text { por la red. }\end{array}$ & $\begin{array}{l}\text { - Sistema CRM - Planificación } \\
\text { del recurso humano necesario } \\
\text { al diseno de la red y su } \\
\text { proyeccion futura. }\end{array}$ \\
\hline & $\begin{array}{l}\text { 12. Sistema de información integrado que vincula a todos los miembros de la } \\
\text { red. con desglose de los datos por sexo. edad. lugar de residencia. origen } \\
\text { étnico y otras variables pertinentes. }\end{array}$ & $\begin{array}{l}\text { - Sistema CRM . Integrar el } \\
\text { sistema informatico con todos } \\
\text { los miembros de la red. }\end{array}$ \\
\hline & 13. Gestión basada en resultados. & $\begin{array}{l}\text { - Cuadro de mando Integral } \\
\text { (CMI) }\end{array}$ \\
\hline 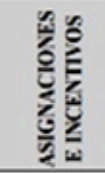 & $\begin{array}{l}\text { 14. Financiamiento adecuado e incentivos financieros alineados con las metas } \\
\text { de la red. }\end{array}$ & $\begin{array}{l}\text { - Plataforma } \\
\text { compras }\end{array}$ \\
\hline
\end{tabular}

Fuente: [13].

\section{Materiales y métodos}

El tipo de investigación es mixto [20,21], porque utiliza fuentes de información cualitativa y cuantitativa. La primera se centra en el: i) análisis de la información documental que desarrollo la investigadora Pulgarín en su tesis de maestría sobre la ESE Oriente (programas y actividades del Plan de Desarrollo Institucional 2012-2015), así como en el análisis de entrevista semiestructurada realizada al gerente de la ESE Oriente 
de Cali, regulada por lo conceptos teóricos de la logística integral (cadena de suministro y score model), y redes integradas de salud; ii) análisis documental del marco legal por el investigador Giraldo, con base en su experiencia en el sector salud.

Y la segunda analiza: i) bases de datos de la Secretaría de Salud Municipal de Cali y de la Alcaldía de la ciudad, comunas 13,14, 15 y 21, en en relación con qué actores participan de manera directa en población, morbilidad, mortalidad, partos, histórico de pacientes atendidos, auditoría general a la Red de Salud Oriente ESE; ii) bases de datos sobre los actores, que se relacionan por la población atendida, la capacidad instalada y el estado de resultados por parte de la ESE Oriente.

El alcance de la investigación parte de la aplicación del análisis de redes [22], utilizando el programa Ucinet 6.0, con el fin de simular y modelar la RIPSS para la red salud del oriente del municipio de Santiago de Cali, teniendo en cuenta los actores regulados y no regulados esbozados en la tabla 1, por estar relacionados de manera directa con la dinámica del territorio abordado.

\section{Resultados}

Los actores que integrarían la RIPSS de la red salud del oriente del municipio de Santiago de Cali son aproximadamente 42 , que se categorizan por naturaleza y área de influencia a partir del análisis de redes [22, $23,24,25]$, como se presenta en la tabla 3 . 
TABLA 3

Actores que integrarían la red integral de prestadores de servicios de salud de la red salud del oriente del municipio de Santiago de Cali

\begin{tabular}{|c|c|c|c|}
\hline $\begin{array}{l}\mathbf{N} . \\
0\end{array}$ & Nombre & Naturaleza & $\begin{array}{c}\text { Área de influencia dentro } \\
\text { de la red }\end{array}$ \\
\hline 1 & Emsanar & \multirow{9}{*}{ EPS } & \multirow{9}{*}{ Comunas $13,14,15$ y 21} \\
\hline 2 & Caprecom & & \\
\hline 3 & Coosalud & & \\
\hline 4 & Selvasalud & & \\
\hline 5 & Calisahud & & \\
\hline 6 & Cafesalud & & \\
\hline 7 & Condor & & \\
\hline 8 & Asmetsalud & & \\
\hline 9 & Ambuq & & \\
\hline 10 & Hospital Carlos Holmes Trujillo & \multirow{25}{*}{ IPS } & Comunas $13,14,15$ y 21 \\
\hline 11 & Centro de Salud El Diamante & & \multirow{9}{*}{ Comuna 13} \\
\hline 12 & Puesto de Salud Calipso & & \\
\hline 13 & Puesto de Salud Comuneros II & & \\
\hline 14 & Puesto de Salud Los Lagos & & \\
\hline 15 & Puesto de Salud Ricardo Bakázar & & \\
\hline 16 & Puesto de Salud Charco Aaul & & \\
\hline 17 & Puesto de Salud Ulpiano Lloreda & & \\
\hline 18 & Puesto de Sahud El Pobhado II & & \\
\hline 19 & Puesto de Salud El Vergel & & \\
\hline 20 & Centro de Salud Marroquin Cauquita & & \multirow{6}{*}{ Comuna 14} \\
\hline 21 & Centro de Salud Manuela Beltrán & & \\
\hline 22 & Puesto de Salud Alirio Mora Beltrán & & \\
\hline 23 & Puesto de Salud Alfonso Bonilla Aragón & & \\
\hline 24 & Puesto de Salud Los Naranjos & & \\
\hline 25 & Puesto de Salud Intervenidas & & \\
\hline 26 & Centro de Salud EI Vallado & & \multirow{5}{*}{ Comuna 15} \\
\hline 27 & Puesto de Salud Comuneros & & \\
\hline 28 & Puesto de Salud Ciudad Córdoba & & \\
\hline 29 & Puesto de Salud Mojica & & \\
\hline 30 & Puesto de Salud El Retiro & & \\
\hline 31 & Centro de Salud Decepaz & & \multirow{4}{*}{ Comuna 21} \\
\hline 32 & Centro de Salud Potrero Grande & & \\
\hline 33 & Puesto de Salud Pizamos & & \\
\hline 34 & Puesto de Salud de Navarro & & \\
\hline 35 & Adolfo Allers & \multirow{5}{*}{$\begin{array}{l}\text { Proveedor } \\
\text { Mayorista }\end{array}$} & \multirow{8}{*}{ Comumas $13,14,15$ y 21} \\
\hline 36 & Droguería Alianza & & \\
\hline 37 & Equipadora Médica & & \\
\hline 38 & Roche & & \\
\hline 39 & LaFrancol & & \\
\hline 40 & Aseo Buritica & \multirow{3}{*}{$\begin{array}{l}\text { Proveedor } \\
\text { Minorista }\end{array}$} & \\
\hline 41 & Institucional S. A. & & \\
\hline 42 & Papelería Andina & & \\
\hline
\end{tabular}


Los actores identificados se organizan en las figuras 4 y 6 , donde el vínculo es el valor 1 y el no vínculo es el valor 0; la primera matriz no aplica el concepto de RIPSS y la segunda sí lo aplica. Posteriormente, se visualiza cada matriz mediante las figuras 5 y 7.

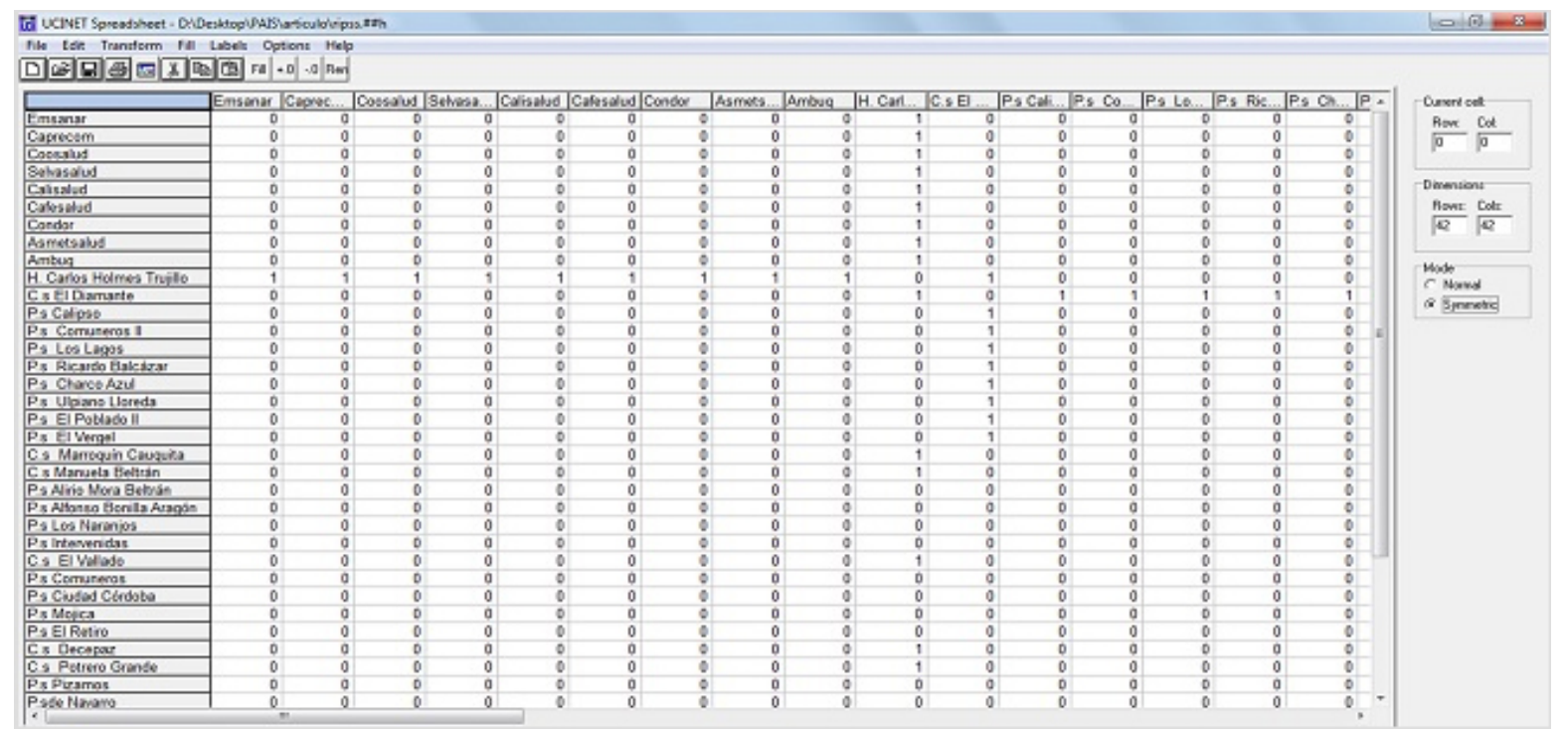

FIGURA 4

Actores de red salud del oriente del municipio de Santiago de Cali Antes de aplicar el concepto de RIPSS Fuente: elaboración propia a partir de Ucinet 6.0.

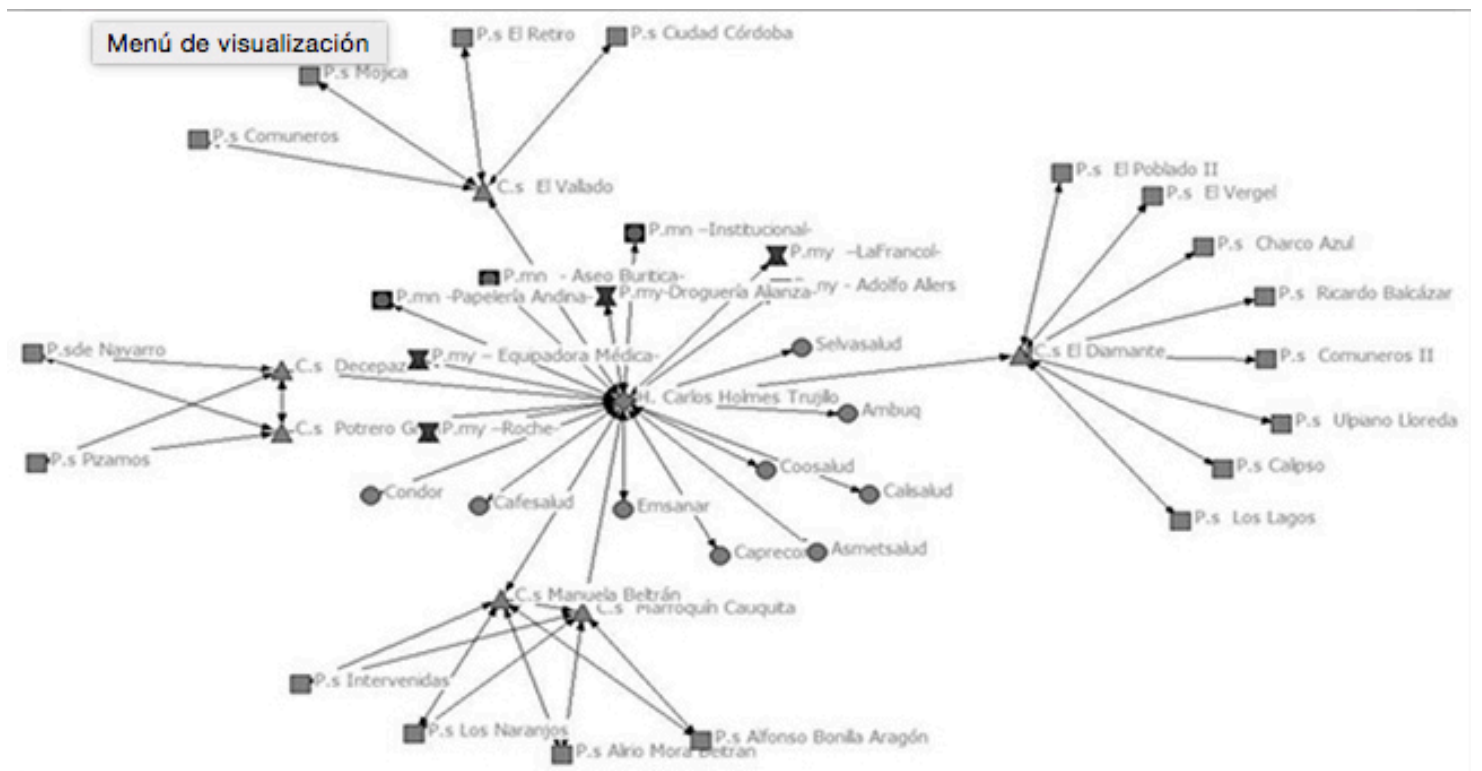

FIGURA 5

Grafo de la red salud del oriente del municipio de Santiago de Cali antes de aplicar la RIPSS Fuente: elaboración propia a partir de Ucinet 6.0. 


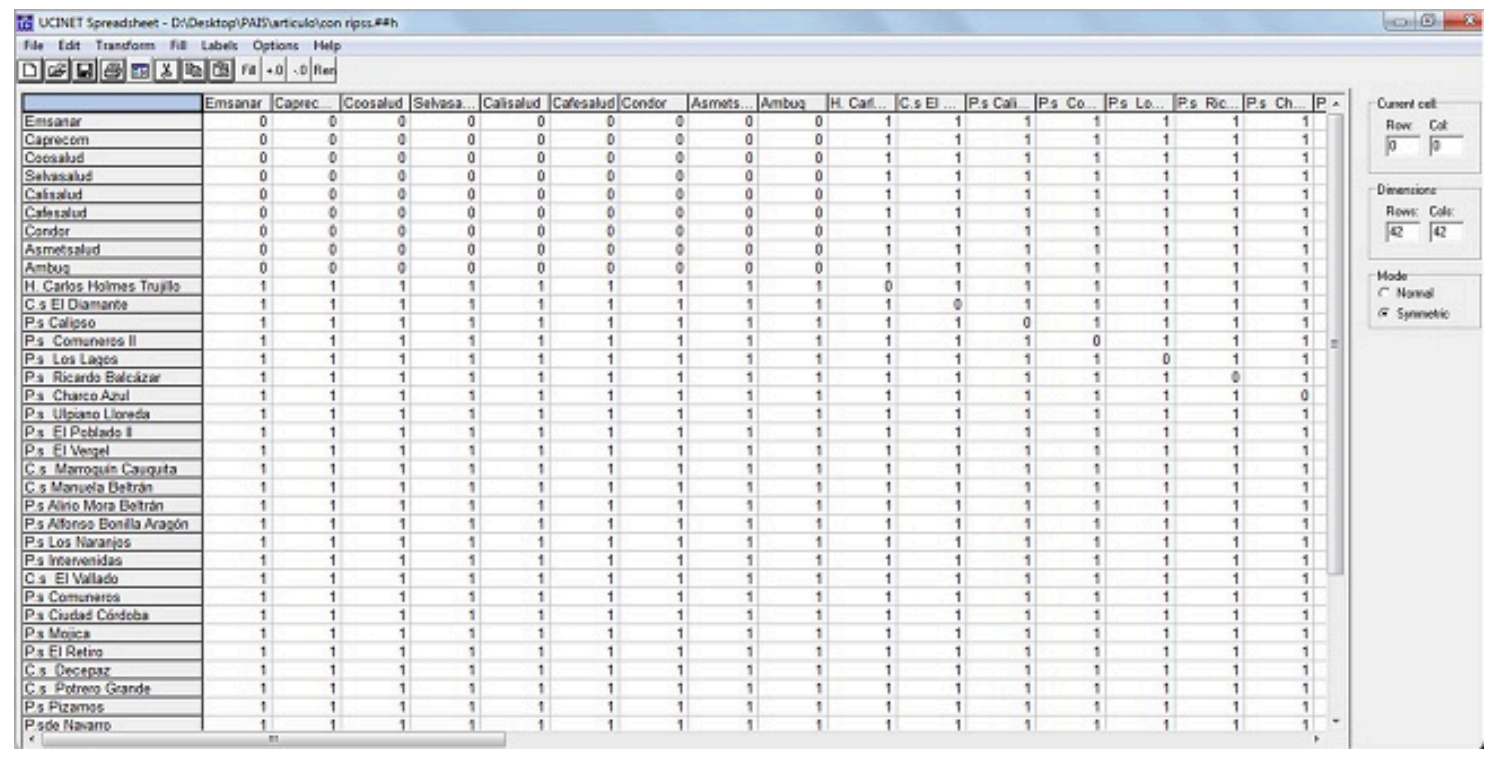

FIGURA 6

Actores de la red salud del oriente del municipio de Santiago de Cali aplicando el concepto de RIPSS con los catorce atributos de la OPS [4] y las herramientas logísticas de Pulgarin [13] Fuente: elaboración propia a partir de Ucinet 6.0.

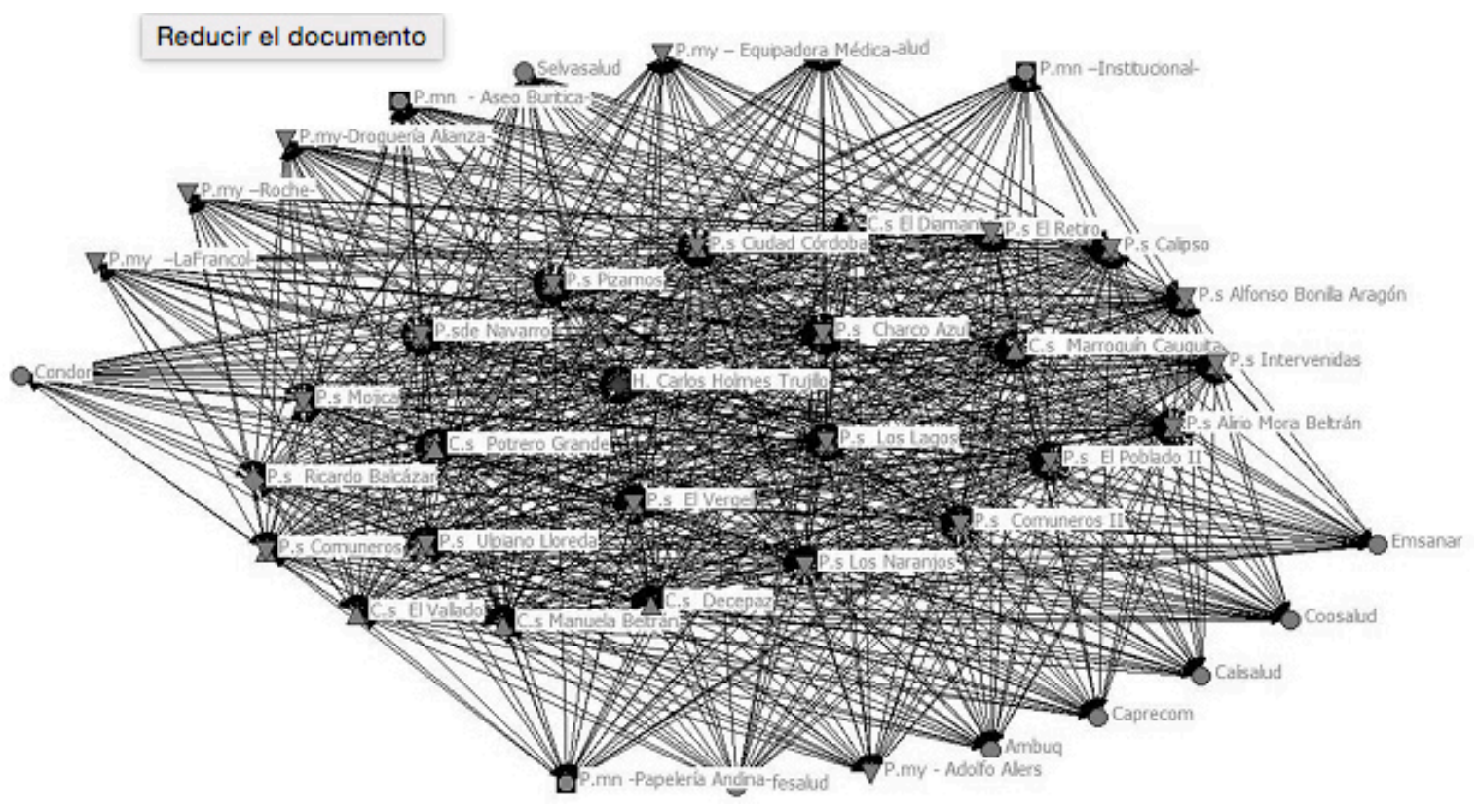

FIGURA 7

Grafo de la red salud del oriente del municipio de Santiago de Cali aplicando el concepto de la RIPSS con los catorce atributos de la OPS [4] y las herramientas logísticas de Pulgarin [13] Fuente: elaboración propia a partir de Ucinet 6.0.

Posteriormente, se realiza el análisis estructural (densidad, indicadores descriptivos media, desviación estándar e índice de centralización) y posicional (grado nodal [degree], grado de intermediación [betweenness], el grado de la cercanía [closenness] si la matriz es simétrica, y si no es simétrica se calcula el Power Bonicint) antes y después de aplicar el concepto de RIPSS, a partir de Tabarquino [22], Lozares [24], Álvarez y Aguilar [26], Kadushin [27], Molina et al [28], Quiroga [29], Wasserman y Faust, [30], Rodríguez [31] y Requena [32]. 
TABLA 4

Análisis estructural y posicional en red de salud del oriente antes y después del concepto de RIPSS ${ }^{\mathrm{i}}$

\begin{tabular}{|c|c|c|c|}
\hline \multicolumn{2}{|c|}{ Red de cadena de suministro de la ESEOriente de Cali } & $\begin{array}{c}\text { Red de salud del oriente } \\
\text { sin apdicar el concepto de } \\
\text { RIPSS }\end{array}$ & \begin{tabular}{|c} 
Red de salud del oriente \\
apdicando el coscepto de \\
RIPSS con los catorce \\
atribetos de la OPS (4) y \\
las herramientas \\
logisticas de Pulgarin \\
(13)
\end{tabular} \\
\hline \multicolumn{4}{|c|}{ Análisis estructural } \\
\hline Nodo o actores & $\begin{array}{l}\text { Representa el número de } \\
\text { personas o grupos u } \\
\text { organizaciones de la red (nxn) }\end{array}$ & \multicolumn{2}{|c|}{42} \\
\hline $\begin{array}{l}\text { Relaciones posibles de la } \\
\text { red }\end{array}$ & $\begin{array}{l}\text { El cálculo de este indicador se } \\
\text { realiza por medio de la formula } \\
\mathrm{Rp}=(\mathrm{nx} \text { (n }-1) \text {, lo cual es el } \\
\text { número total de nodos por el } \\
\text { número total de nodos menos } \\
\text { uno }\end{array}$ & \multicolumn{2}{|c|}{1722} \\
\hline \multicolumn{4}{|c|}{$\begin{array}{l}\text { El cálculo de este indicador se } \\
\text { realiza sumando el total de filas } \\
\text { o de columnas }\end{array}$} \\
\hline Densidad de la red & $\begin{array}{l}\text { El cálculo de este indicador se } \\
\text { realiza por medio de la formula } \\
\text { D- (Re/Rp) xl00), lo cual es el } \\
\text { número de relaciones } \\
\text { existentes dividido entre las } \\
\text { posibles y multiplicado por } \\
100 \text {. Permite conocer la alta o } \\
\text { baja conectividad de la red }\end{array}$ & $\begin{array}{l}5.7 \% \text {, la conectividad } \\
\text { entre los actores de la red } \\
\text { es baja }\end{array}$ & $\begin{array}{l}84: 2 \%, \text { la conectividad } \\
\text { entre los actores de la red } \\
\text { es alta }\end{array}$ \\
\hline $\begin{array}{l}\text { La media de todas las } \\
\text { relaciones }\end{array}$ & $\begin{array}{l}\text { La media indica el número } \\
\text { promedio de conexiones que } \\
\text { tienen los actores en la rod }\end{array}$ & 2.3 & 34 \\
\hline La desviación estándar & $\begin{array}{l}\text { La desviación indica la } \\
\text { dispersión entre los actores }\end{array}$ & 3.6 & 7 \\
\hline
\end{tabular}

Fuente: elaboración propia a partir de Ucinet 6.0 
TABLA 4 (CONT.)

Análisis estructural y posicional en red de salud del oriente antes y después del concepto de RIPSS ${ }^{\mathrm{i}}$

\begin{tabular}{|c|c|c|c|}
\hline \multicolumn{4}{|c|}{ Analisis posicional } \\
\hline $\begin{array}{l}\text { E network centralization } \\
\text { o indice de centralizacion }\end{array}$ & $\begin{array}{l}\text { El nefwork centralization o } \\
\text { indice de centralización mide si } \\
\text { la red se comporta como una } \\
\text { red estrella, es decir, si existe } \\
\text { un solo actor central en la red } \\
\text { IC }=100 \% \text {, red estrella } \\
\text { IC- } 0 \% \text {, red honizontal }\end{array}$ & $\begin{array}{l}52.93 \% \text {, esto indica que la } \\
\text { red está cerca de no } \\
\text { comportarse como una red } \\
\text { estrella, donde un actor } \\
\text { desermpeña un papel } \\
\text { central que controla a toda } \\
\text { la red }\end{array}$ & $\begin{array}{l}16.59 \% \text {, esto indica que } \\
\text { la red está cerca de no } \\
\text { comportarse como una } \\
\text { red estrella, donde un } \\
\text { actor desempeña un } \\
\text { papel central que } \\
\text { controla a toda la red }\end{array}$ \\
\hline $\begin{array}{l}\text { Rango o el grado nodal } \\
\text { (degree) }\end{array}$ & $\begin{array}{l}\text { El indicador calkulado, } \\
\text { denominado rango, permite } \\
\text { evidenciar quién es el actor o } \\
\text { quienes son los actores } \\
\text { centrales en la red }\end{array}$ & $\begin{array}{l}\text { E actor central es el } \\
\text { hospital Carlos Holmes } \\
\text { Trujillo }(23) \text {, con un } 56 \% \\
\text { de conectividad en la red }\end{array}$ & $\begin{array}{l}\text { Los actores centrales de } \\
\text { la red son el hos pital, } \\
\text { centros y puestos de } \\
\text { salud ( } 41 \text { ), con un } 100 \% \\
\text { de conectividad en la red }\end{array}$ \\
\hline $\begin{array}{l}\text { Egrado de intermediación } \\
\text { (berweenness) }\end{array}$ & $\begin{array}{l}\text { Esboza cuál es cl actor de } \\
\text { mayor intermediación en la red, } \\
\text { es decir, que cxiste un actor } \\
\text { que actúa como nodo central y } \\
\text { actor puente }\end{array}$ & $\begin{array}{l}\text { El actor de mayor } \\
\text { intemediación en la red es } \\
\text { el hospital Carlos Holmes } \\
\text { Trujillo (753), es decir, que } \\
\text { es el nodo central y el } \\
\text { actor puente de la red }\end{array}$ & $\begin{array}{l}\text { Los actores de mayor } \\
\text { intermediación en la red } \\
\text { son el hospital, los } \\
\text { centros y puestos de } \\
\text { salud ( } 5.44) \text {, es decir, que } \\
\text { son los nodos centrales } \\
\text { y actores puente de la rec }\end{array}$ \\
\hline $\begin{array}{l}\text { El grado de cercania } \\
\text { (closenness) en la red se } \\
\text { calcula ya que la matriz es } \\
\text { simctrica }\end{array}$ & $\begin{array}{l}\text { Este indicador se calcula si la } \\
\text { matriz es simetrica, señala qué } \\
\text { actor tiene mis cercania con } \\
\text { los otros actores de la red }\end{array}$ & $\begin{array}{l}\text { E actor con mayor grado } \\
\text { de crecania es el hospital } \\
\text { Carlos Holmes Trujillo (59) }\end{array}$ & $\begin{array}{l}\text { Los actores con mayor } \\
\text { grado de crecania son el } \\
\text { hospital, los centros y } \\
\text { puestos de salud (41) }\end{array}$ \\
\hline
\end{tabular}

Fuente: elaboración propia a partir de Ucinet 6.0

\section{Hallazgos}

La matriz 1 y la figura 1 evidencian que el actor central en la red de salud del oriente del municipio de Santiago de Cali, antes de aplicar el esquema de RIPSS, es el hospital Carlos Holmes Trujillo, por la cadena de suministro y contratación, debido a que se encarga de establecer las compras y distribución en centros y puestos de salud en el área de influencia de la zona oriente de Cali.

La matriz 2 y la figura 2 evidencian que la aplicación del esquema y concepto de la RIPSS, con los catorce atributos de la OPS [4] y las herramientas logísticas propuestas por Pulgarin [13], a la red de salud del oriente del municipio de Santiago de Cali, modifica las relaciones de la red inicial, en cuanto a que: i) los puestos de salud y centros de salud adquieren relevancia, por la conectividad establecida con las EPS y el hospital Carlos Holmes Trujillo, es decir, que se dinamizan en varios nodos y con ello incrementan la efectividad en la prestación del servicio; los usuarios pueden desplazarse en diferentes puntos y optimizar la infraestructura de la red; ii) se obliga al desarrollo adecuado de un sistema de información y plataforma eficaz que debe estar en la nube, donde los actores de la RIPSS tendrán acceso a esta; iii) se aplica la herramienta de la telemedicina, como conectividad y acceso a centros de salud y puestos de salud para servicios especializados para la población. 
La tabla 4 esboza un cambio considerable al aplicar el esquema y concepto de la RIPSS a la red de salud del oriente, en el sentido de: i) incremento de la conectividad de la red en un $78.5 \%$, pasando de un 5.7 $\%$ a un $84.2 \%$; ii) potencialidad por gobernanza y eficiencia de la red en logística y toma de decisiones de forma horizontal y no jerárquica, gracias al incremento de las relaciones existentes de 98 a 1450, la media de las relaciones de 2.3 a 34, y de pasar de un actor a tres actores centrales; iii) disminución del índice de centralización en $36.34 \%$, pasando de $52.93 \%$ a $16.59 \%$, lo cual refleja más interrelación entre actores de la red; iv) incremento de un actor a tres actores con intermediación y cercanía en la red.

\section{Discusión}

La aplicación de un esquema de RIPSS en el contexto colombiano debe tener en cuenta la existencia de los proveedores en las relaciones formales de la estructura en red, por su capacidad de transacción y los recursos económicos que no poseen otros actores.

A pesar de lo novedoso que resulta este nuevo esquema en materia logística y de administración de salud, no reemplaza la institucionalidad de las entidades públicas, por su aprendizaje organizacional y social en el territorio, a pesar de que el modelo jerarquice las EPS. Estas siguen una lógica de maximización de la utilidad y no de prioridad de la población en el territorio abarcado.

Las RIPSS mejoran el flujo de información y el reconocimiento entre actores, pero por ser una regulación y esquema nuevo pueden generar asimetrías, no en la información que fluye a través de la red, sino en los protocoles de cooperación entre las EPS y las IPS, hospitales, centros de salud, puestos de salud, clínicas y laboratorios.

\section{Conclusiones}

El análisis cuantitativo de redes evidencia que el concepto y esquema de RIPSS, aplicado a la red de salud del oriente del municipio de Santiago de Cali, establece una mayor optimización por el aumento en la conectividad e interrelación entre actores regulados (EPS e IPS) y no regulados (proveedores mayoristas y minoristas), lo cual obliga a incorporar instrumentos de logística integral para validar la gestión de salud en tiempos, escalas y resultados. De lo contrario, se aplican redes de atención en servicios de salud fragmentadas o parcialmente integradas.

Las RIPSS aportan al componente primario y complementario de prestación del sector de la salud, interdependencia, interrelación y coordinación de actores públicos, privados y mixtos, en un territorio que puede ser exitoso, con la incorporación de tecnología y competencias especializadas para enfermedades, servicios de complejidad y pertinencia e incidencia sociocultural en el territorio abarcado e intervenido.

El modelo de RIPSS mejora la conectividad en una red de salud, pero disocia la responsabilidad jerárquica en la prestación del servicio público de salud, ratificando con ello la mejora en las zonas de no mercado (logística y costos de transacción), es decir, que incrementa la gobernanza en el sector de la salud en cuanto a la promoción y la mediación por parte de las entidades estatales.

\section{Agradecimientos}

Artículo de investigación, producto de la aplicación del enfoque teórico y metodológico del análisis de redes llevado a cabo en la tesis doctoral en Administración de la Universidad del Valle (Cali, Colombia) por el profesor Tabarquino, titulada La red de política pública de regulación del servicio público de las tecnologías de las información y las comunicaciones (TIC) en Colombia 2009-2016: el acceso (universalidad) y la equidad como principios rectores, bajo la dirección del profesor Edgar Varela Barrios; y de los aportes de la tesis del año 2015, 
titulada Diseño de una red integrada de servicios de salud basada en la gestión de la cadena de suministro: caso de tres organizaciones de salud de la ciudad de Santiago De Cali, desarrollada por la investigadora Pulgarín bajo la tutoría del profesor Arley Torres, en el programa de Maestría en Logística Integral de la Universidad Autónoma de Occidente, Cali, Colombia; y el análisis del marco regulatorio de las redes integrales de prestadores de servicios de salud (RIPSS) por el docente e investigador Giraldo por su amplia experiencia en administración y gerencia del sector salud.

\section{Referencias}

1. Ministerio de Salud y Protección Social de Colombia. Resolución 1441 del 21 de abril del 2016 por la cual se establecen los estándares, criterios y procedimientos para la habilitación de las Redes Integrales de Prestadores de Servicios de Salud y se dictan otras disposiciones. Disponible en: http://achc.org.co/documentos/prensa/res-1 44116\%20habilitacion\%20redes\%20integradas\%20ips.pdf

2. Mendes EV. Las redes de atención de salud. Brasilia: Organización Panamericana de la Salud; 2013. Disponible en: http://apps.who.int/iris/bitstream/10665/173997/1/LAS-REDES-DE-ATENCION-DE-SALUD-web3 $\% 5$ B1\%5D.pdf

3. Organización Panamericana de la Salud (OPS). La renovación de la atención primaria en salud en las Américas. Documento de posición de la Organización Panamericana de la Salud/Organización Mundial de la Salud (OPS/ OMS); 2007. Disponible en: https://cursos.campusvirtualsp.org/file.php/118/Modulo_I/md3-lp-renovacion -APS-spa-2007.pdf

4. Organización Panamericana de la Salud (OPS). La renovación de la Atención Primaria en Salud en la Américas. Redes integradas de servicios de salud - conceptos, opciones de política y hoja de ruta para su implementación en las Américas; 2010. Disponible en: http://www.paho.org/uru/index.php?option=com_docman\&view=download\&category_slug=publicacio nes-sistemas-y-servicios-de-salud\&alias=145-redes-integradas-de-servicios-de-salud-aps-n4\&Itemid=307

5. Artaza O, Méndez C, Holder R, Suárez JM. Redes integradas de servicios de salud: el desafío de los hospitales. Documentos OPS/OMS en Chile No. 1. Santiago, Chile: OPS/OMS; 2011. Disponible en: http://www1.pah o.org/chi/images/PDFs/redes_integrales_de_servicios.pdf

6. Barragán Bechara JC, Riaño Casallas MI, Martínez, M. Redes integradas de servicios de salud: hacia la construcción de un concepto. Revista Universidad y Salud. 2012; 14(2):186-96. Disponible en: http://www.scielo.org.co/sci elo.php?script=sci_arttext\&pid=S0124-71072012000200008

7. Balladelli PP. Redes integradas de servicios de salud y atención en salud mental. Ponencia presentada en la Asamblea Provincial del Capítulo 2010-2014 de la Orden Hospitalaria de San Juan de Dios. Buenos Aires, Argentina, 2013. Disponible en: http://hsjd.org/asamblea2013/docs/balladelli_redes_integradas.pdf

8. Eraso N. Las redes integradas de servicios de salud: una propuesta en construcción. Bogotá: Universidad del Rosario, Escuela de Medicina y Ciencias de la Salud, Documentos de investigación N.o 14; 2011. Disponible en: http:// www.urosario.edu.co/urosario_files/ab/abe56836-f233-459d-bc20-f5f29bf89ed3.pdf

9. Gillies R, et al. Conceptualizing and measuring integration: Findings from the health systems integration study. Hospital and Health Services Administration. 1993; 38(4):467-89. Disponible en: http://journals.lww.com/jh monline/Abstract/1993/10000/Conceptualizing_and_Measuring_Integration_.3.aspx

10. Lau S, Soler S. Las redes integradas de servicios de salud desde la realidad cubana. Revista Cubana de Salud Pública. 2009; 35(4).Disponible en: http://scielo.sld.cu/scielo.php?script=sci_arttext\&pid=S0864-346620090004000 05

11. Ocampo-Rodríguez MV, Urrutia VFB, Montoya-Rojas JP, Bautista-Botton DC. Sistemas y modelos de salud, su incidencia en las redes integradas de servicios de salud. Revista Gerencia y Políticas de Salud. 2013; 12(24):114-29. Disponible en: http://www.scielo.org.co/pdf/rgps/v12n24/v12n24a08.pdf 
12. Provan K, Milward B. Do networks really work? A framework for evaluating public-sector organizational networks. Public Administration Review. 2001; 61(4):414-23. Disponible en: http://wiki.dbast.com/images/a/a5/Do_N etworks_Really_Work_-_A_Framework_for_Evaluating_Public-Sector_Organizational_Networks.pdf

13. Pulgarín Henao DM. Diseño de una red integrada de servicios de salud basada en la gestión de la cadena de suministro: caso de tres organizaciones de salud de la ciudad de Santiago de Cali [tesis de maestría]. Programa de Logística Integral, Universidad Autónoma de Occidente, Cali, Colombia; 2015. Disponible en: https://red.ua o.edu.co/bitstream/10614/7883/1/T05881.pdf

14. Pulgarín Henao DM, Tabarquino Muñoz RA. Análisis del diseño de una red integrada de servicios de salud. Revista Semestre Económico. 2016. Disponible en: http://www.scielo.org.co/scielo.php?pid=S012063462016000200 $175 \&$ script $=$ sci_abstract\&tlng=es

15. Vásquez ML, Vargas I. Redes integradas de servicios de salud: ¿Solución o problema? Revista Ciencias de la Salud. 2006; 4(1):5-9. Disponible en: http://www.scielo.org.co/pdf/recis/v4n1/v4n1a1.pdf

16. Congreso de Colombia. Ley 1438 del 19 de enero del 2011, por medio de la cual se reforma el Sistema General de Seguridad Social en Salud y se dictan otras disposiciones. Disponible en: https://www.minsalud.gov.co/Norm atividad_Nuevo/LEY\%201438\%20DE\%202011.pdf

17. Congreso de Colombia. Ley Estatutaria 1751 del 16 de febrero del 2015, por medio de la cual se regula el derecho fundamental a la salud y se dictan otras disposiciones. Disponible en: https://www.minsalud.gov.co/Normativ idad_Nuevo/Ley\%201751\%20de\%202015.pdf

18. Ministerio de Salud y Protección Social de Colombia. Resolución 429 del 17 de febrero de 2016, por medio de la cual se adopta la Política de Atención Integral en Salud. Disponible en: https://www.minsalud.gov.co/Normat ividad_Nuevo/Resoluci\%C3\%B3n\%200429\%20de\%202016.pdf

19. Ministerio de Salud y Protección Social de Colombia. Política de Atención Integral en Salud. Bogotá: autor; 2016. Disponible en: https://www.minsalud.gov.co/sites/rid/Lists/BibliotecaDigital/RIDE/DE/modelo-pais-2016. pdf

20. Eisenhardt KM. Building theories from case study research. Academy of Management Review. 1989; 14(4):532-50. Disponible en: https://www.business.illinois.edu/josephm/BADM504_Fall\%202016/Eisenhardt1989.pdf

21. Patton MQ. Qualitative research and evaluation methods. Thousand Oaks, CA: Sage; 2002. Disponible en: https ://us.sagepub.com/en-us/nam/qualitative-research-evaluation-methods/book232962

22. Tabarquino Muñoz RA. El análisis organizacional y de política pública a partir del enfoque de redes. Revista Tendencias. 2016; XVII(2). Disponible en: http://revistas.udenar.edu.co/index.php/rtend/article/view/2853/ 3209

23. Hanneman RA. Introducción a los métodos del análisis de redes sociales. 2001. Disponible en: http://revista-red es.rediris.es/webredes/textos/Introduc.pdf

24. Lozares C. Bases socio-metodológicas para el análisis de redes sociales. Empiria. Revista de Metodología de Ciencias Sociales. 2005; (10):9-35. Disponible en: http://revistas.uned.es/index.php/empiria/article/view/1042

25. Verd Pericas JM, Marti Olive J. Muestreo y recogida de datos en el análisis de redes sociales. Revista Questiio. 1999; 23(3):507-24. Disponible en: http://upcommons.upc.edu/bitstream/handle/2099/4120/article.pdf?sequence $=4 \&$ isAllowed $=\mathrm{y}$

26. Álvarez Velásquez A, Aguilar Gallegos N. Manual introductorio de análisis de redes sociales. Centro de Capacitación y Evaluación para el Desarrollo Rural. Universidad Autónoma del estado de México. México: Internacional Network for Social Network Análisis; 2005.

27. Kadushin C. Comprender las redes sociales: teorías, conceptos y hallazgos. CIS-Centro de Investigaciones Sociológicas; 2013.

28. Molina JL, Quiroga A, Martí, Maya I, De Federico A. Talleres de autoformación con programas informáticos de análisis de redes sociales. Barcelona: Universitat Autònoma de Barcelona, Servei de Publicacions, Bellaterra; 2006. Disponible en: https://ddd.uab.cat/pub/llibres/2006/140680/talaupro_a2006iSPA.pdf

29. Quiroga A. Introducción al análisis de datos reticulares. Revista Hispana para el Análisis de Redes Sociales. 2003. Disponible en: http://revista-redes.rediris.es/webredes/talleres/redes_avanzado.pdf?q=anlisis-de-algoritmos 
30. Wasserman S, Faust K. Análisis de redes sociales. Métodos y aplicaciones. CIS-Centro de Investigaciones Sociológicas; 2013.

31. Rodríguez J. Análisis estructural y de redes. CIS-Centro de Investigaciones Sociológicas; 2005.

32. Requena Santos F. Análisis de redes sociales: orígenes, teorías y aplicaciones. CIS-Centro de Investigaciones Sociológicas; 2003.

\section{Notas}

i Las relaciones existentes se han calculado multiplicando el número total de nodos por el número total de nodos menos uno $42 \times(42-1)=1722$.

* Artículo de investigación.

\section{Licencia Creative Commons CC BY 4.0}

Como citar este artículo: Tabarquino Muñoz RA, Pulgarin Henao DM, Giraldo Varón AF. Dinámica de una red integral de prestadores de servicios de salud (RIPSS). Rev Gerenc Polít Salud. 2017; 17(34): 1-17. https://doi.org/10.11144/Javeriana.rgps17-34.drip 